\title{
A New Microscopic Calculation for the Uniform Electron Fluid
}

\author{
A. Bouchebak ${ }^{a}$, M.K. Al-Sugheir ${ }^{b, *}$ And H.B. Ghassib ${ }^{c}$ \\ ${ }^{a}$ Department of Physics, Ecole Normale Superieure, Kouba, Algiers, Algeria \\ ${ }^{b}$ Department of Physics, The Hashemite University, Zarqa, Jordan \\ ${ }^{c}$ Department of Physics, The University of Jordan, Amman, Jordan
}

(Received October 6, 2010; in final form November 23, 2010)

\begin{abstract}
The static fluctuation approximation is applied for the first time to an electronic system. A simple model (a uniform electron fluid) is chosen to explore the applicability of static fluctuation approximation to electrons in metals. The thermodynamic properties - the internal energy per particle, the pressure, the entropy per unit volume, the heat capacity per unit volume, and the chemical potential - are calculated over a wide range of densities within the metallic-density region. Finally, the pair-correlation function for the electron fluid is evaluated. Values of this function are then tabulated for zero-interparticle separation at several densities of interest. The results of this work are found to be in good agreement with several other many-body calculations.
\end{abstract}

PACS: 05.30.Fk, 71.10.Ay, 71.10.Ca

\section{Introduction}

The hypothetical uniform electron fluid consists of a large number $N$ of electrons embedded in a uniform background of positive charge chosen to ensure overall charge neutrality. Since we are interested only in bulk properties, the system is studied in the thermodynamic limit: $N \rightarrow \infty$ and $\Omega \rightarrow \infty$, in such a way that the number density $n \equiv N / \Omega$ remains constant ( $\Omega$ being the [normalization] volume). This implies that surface effects are neglected [1]. The validity of the thermodynamic limit in such a system has been rigorously proven [2]. As a model for the conduction electrons in a solid, where the lattice of the positive ions is replaced with an equivalent uniform medium, this leads to enormous simplifications. In particular, the ionic charge density fluctuations and, therefore, the interactions of the electrons with the lattice vibrations are ignored.

In the absence of spin polarization, the model is fully specified by two dimensionless parameters: the density parameter (the Wigner-Seitz radius) $r_{\mathrm{s}}=a / a_{0}$, which is defined as the average separation $a$ between electrons measured in units of the effective Bohr radius $a_{0} \equiv \hbar^{2} / m e^{2}$, and the coupling-parameter $\Gamma=E_{c} / E_{p}$, which is the relative strength between the Coulomb and kinetic energies, $E_{c}$ and $E_{p}$, respectively. Depending on the parameter $\Gamma$, the electron fluid may exhibit three different regimes in terms of $r_{\mathrm{s}}$ : the weak $\left(r_{\mathrm{s}} \leq 1\right)$, intermediate $\left(1 \leq r_{\mathrm{s}} \leq 10\right)$, and strong $\left(r_{\mathrm{s}} \geq 10\right)$ Coulomb coupling regimes [3]. The distinction between low and high densities is often quantified by the parameter $r_{\mathrm{s}}$

* corresponding author; e-mail: msugh@hu.edu.jo which is related to the electron density by $4 \pi\left(a_{0} r_{\mathrm{s}}\right)^{3} / 3=$ $\Omega / N \equiv n^{-1}$. At small $r_{\mathrm{s}}$ (high density), the electrons form a weakly-coupled Fermi liquid, while at large $r_{\mathrm{s}}$ (low density) they are expected, as Wigner first predicted, to undergo a freezing transition forming a crystalline phase known as the Wigner crystal: the electrons arrange themselves in the configuration of lowest potential energy, which is always greater than (or equal to) the exact energy [4]. The bcc lattice was shown to be the optimal configuration, in agreement with classical arguments by Wigner [5].

The lack of a comprehensive microscopic theory for the electronic system has motivated us to revisit it. This is the first time the static fluctuation approximation (SFA) is applied to an electronic system. A simple model (a uniform electron fluid) is chosen to explore the validity of SFA in such systems. The model is used as a reference system in most realistic calculations of electronic structure in condensed matter.

In real solids, the compensating background of positive charge is neither a rigid nor a uniform charge distribution; rather, it is discrete and deformable. Specifically, the lattice ions form positive charge concentrations localized at the lattice sites; these perform small oscillations about their equilibrium positions, generating phonons. Naturally this influences the electron distribution. By virtue of their interaction potential with the conduction electrons, the ions induce a polarization of the electron cloud which in turn affects the ionic motion [6-9]. It is well known that macroscopic systems exhibit collective behavior. Of particular interest here are: (a) electronic oscillations, which are known as plasmons; and (b) motion of the ions, which is harmonic (phonons); but these phonons have relatively high energy and do not con- 
tribute much to the low-temperature properties of the system. Because the ion cores (formed by nuclei and the inner electrons) are much more massive than the electrons, their characteristic velocity is much lower. The electrons generally react so quickly that the ion cores can be considered stationary and the electron density has its equilibrium value at all times. Also, the collective description of the system should incorporate the electronelectron interaction caused by the virtual exchange of phonons and the effects of the periodic potential of the crystal ions which have important consequences for the dynamics of the electrons.

Screening is one of the most important manifestations of many-body effects in metals, semiconductors and plasmas. It simply means that the interaction between two particles is modified by the surrounding particles. The motion of an electron in a system depends on that of all other electrons in the system. The electronic interaction causes a Coulomb hole, form around each electron. Electron and hole behave as a single object, namely, a quasiparticle. These quasiparticles interact with each other through a weak screened Coulomb potential. The simplest example of this kind, based on the Thomas-Fermi approximation, is the response of the system to the presence of an impurity atom in a metal, which involves the rearrangement of its charge distribution. In this case, the static screened Coulomb interaction has formally the spatial dependence of the Yukawa potential [1, 10].

A great variety of techniques have been developed for tackling the electron fluid, although each of these is known to be plagued by a number of shortcomings. Two fundamentally different types of approach have been considered. The first category looks into the behavior of the system in momentum space and the calculations are carried out self-consistently, such as the random phase approximation (RPA) [11] and the theory of Singwi and co-workers (STLS) [12]. The second category studies numerically the system in direct space, and is based on the analysis of a subsystem containing a small number of particles, such as the variational and diffusion Monte Carlo methods $[3,13,14]$, the variational Fermi-hypernetted-chain technique $[15,16]$, the coupled-cluster expansion $[17,18]$, and numerical many-body perturbation expansion [19]. All these techniques have their strengths as well as limitations. Some of them rely heavily on extensive computations; but none technique is likely to provide the answers to all the questions we have about this system.

The aim of the present work is to calculate the thermodynamic properties as well as the pair-correlation function of the electron fluid using the so-called SFA [20, 21] with the static screened Coulomb potential. The main advantage of this non-Green-function method is its relative simplicity. In particular, the temperature enters as in ordinary quantum statistical mechanics.

In a series of papers, SFA was developed for calculating the properties of, among other systems, liquid ${ }^{4} \mathrm{He}[21]$, liquid ${ }^{3} \mathrm{He}[22]$, and spin-polarized atomic hy- drogen [23]. More recently, SFA was also applied successfully to hot nuclear matter [24]. The results of these calculations were in qualitative and quantitative agreement with other many-body methods. The theory contains no adjustable parameters and the only approximation is that the square of the local-field operator is replaced with its mean value. The physical implication is that the true quantum-mechanical spectrum of this operator is replaced with a distribution around its expectation value.

The outline of this paper is as follows: In Sect. 2 we present our SFA formalism and theoretical framework. The thermodynamic properties as well as the pair-correlation for the electron fluid are given in Sect. 3. Finally, in Sect. 4, we present the results and make some concluding remarks.

\section{Theoretical framework}

\subsection{The many-body Hamiltonian}

The system of $N$ degenerate interacting electrons moving in a uniform background of neutralizing positive charge is described by the Hamiltonian [1]:

$$
\hat{H}=\hat{H}_{\mathrm{el}}+\hat{H}_{\mathrm{b}}+\hat{H}_{\mathrm{el}-\mathrm{b}},
$$

where $\hat{H}_{\mathrm{el}}, \hat{H}_{\mathrm{b}}$ and $\hat{H}_{\mathrm{el}-\mathrm{b}}$ are the electron-electron, background-background (positive ions) and electronbackground Hamiltonians, respectively.

Since the background is rigid and uniform, the ionic parts of $\hat{H}$ can readily be simplified as [1]:

$$
\begin{aligned}
& \hat{H}_{\mathrm{b}}+\hat{H}_{\mathrm{el}-\mathrm{b}}=-\frac{e^{2} N^{2}}{2 \Omega}\left(\frac{4 \pi}{q_{\mathrm{TF}}^{2}}\right), \\
& q_{\mathrm{TF}}=\left(4 q_{\mathrm{F}} / \pi a_{0}\right)^{1 / 2} .
\end{aligned}
$$

Here $q_{\mathrm{TF}}$ is the Thomas-Fermi wave number and $q_{\mathrm{F}} \equiv$ $\left(3 \pi^{2} n\right)^{1 / 3}$ is the Fermi wave number; $q_{\mathrm{TF}}^{-1}$ is a measure of the screening length.

The dynamic part of the total Hamiltonian can be written as

$$
\begin{aligned}
& \hat{H}_{\mathrm{el}}=\int \mathrm{d}^{3} \boldsymbol{r} \hat{\Psi}^{+}(\boldsymbol{r})\left(-\frac{\hbar^{2} \nabla^{2}}{2 m}\right) \hat{\Psi}(\boldsymbol{r}) \\
& +\frac{1}{2} \iint \mathrm{d}^{3} \boldsymbol{r}_{1} \mathrm{~d}^{3} \boldsymbol{r}_{2} \\
& \quad \times \hat{\Psi}^{+}\left(\boldsymbol{r}_{1}\right) \hat{\Psi}^{+}\left(\boldsymbol{r}_{2}\right) V(\boldsymbol{r}) \hat{\Psi}\left(\boldsymbol{r}_{1}\right) \hat{\Psi}\left(\boldsymbol{r}_{2}\right),
\end{aligned}
$$

where the first term is the kinetic energy of the electrons and the second term represents the mutual interaction between a pair of electrons described by the two coordinates $\boldsymbol{r}_{1}$ and $\boldsymbol{r}_{2}$. The operators $\hat{\Psi}^{+}(\boldsymbol{r})$ and $\hat{\Psi}(\boldsymbol{r})$ are, respectively, the creation and annihilation field operators that satisfy the fermion anti-commutation relations.

The static screened Coulomb potential has formally the spatial-dependence of the Yukawa potential $[1,10]$ :

$$
V(r)=\left(e^{2} / r\right) \mathrm{e}^{-q_{\mathrm{TF}} r} .
$$

Thus, the simple spin-independent Coulomb law between two electrons $V(r)=e^{2} / r$, is "shielded" with the 
Thomas-Fermi (TF) screening length $q_{\mathrm{TF}}^{-1}$. In a uniform electron fluid, all physical properties must be invariant under spatial translations and rotations. This implies that $V(r)$ depends only on the relative interparticle separation $\left|\boldsymbol{r}_{1}-\boldsymbol{r}_{2}\right|$, and the single-particle wavefunction is a plane wave

$$
\psi_{\boldsymbol{k} \sigma}(\boldsymbol{r})=\frac{1}{\sqrt{\Omega}} \mathrm{e}^{-\mathrm{i} \boldsymbol{k} \cdot \boldsymbol{r}} \eta_{\sigma},
$$

where $\boldsymbol{k}$ represents the wavevector, and $\eta_{\sigma}$ can be either the spin-up or spin-down wavefunction.

Since the TF approach relies on a purely static description, all dynamic effects are washed out from the start; in particular, those responsible for the algebraic tails $[25,26]$. This is just a first step in applying SFA to the electron fluid. A more realistic interaction will be used in future works.

In the notation of second quantization, $\hat{H}$ can be written as

$$
\begin{aligned}
\hat{H} & =\sum_{\boldsymbol{k} \sigma} \frac{\hbar^{2} k^{2}}{2 m} \hat{a}_{\boldsymbol{k} \sigma}^{+} a_{\boldsymbol{k} \sigma} \\
& +\frac{1}{2 \Omega} \sum_{\sigma \lambda} \sum_{\boldsymbol{k} \boldsymbol{p}, \boldsymbol{q} \neq 0} V(q) \hat{a}_{\boldsymbol{k}+\boldsymbol{q}, \sigma}^{+} \hat{a}_{\boldsymbol{p}-\boldsymbol{q}, \lambda}^{+} a_{\boldsymbol{p} \lambda} a_{\boldsymbol{k} \sigma},
\end{aligned}
$$

$V(q)$ being the Fourier transform of the pair potential defined by

$$
V(q)=\int \mathrm{d}^{3} r V(r) \exp (-\mathrm{i} \boldsymbol{q} \cdot \boldsymbol{r})=4 \pi e^{2} /\left(q^{2}+q_{\mathrm{TF}}^{2}\right) .
$$

The exclusion of $\boldsymbol{q}=0$ in the Hamiltonian serves to subtract the positive background.

\subsubsection{Basic equations}

\subsection{SFA formalism}

The formalism on which our computational procedure is based (SFA) has been fully described in the literature $[21,22]$. For convenience, a summary is given here to introduce the notation as well as some equations necessary for studying the system.

In the Heisenberg picture, the time evolution of any operator $\hat{a}_{\boldsymbol{k}}^{+}$is given by

$$
\hat{a}_{\boldsymbol{k}}^{+}(\tau)=\exp (\tau \hat{H}) \hat{a}_{\boldsymbol{k}}^{+}(0) \exp (-\tau \hat{H}),
$$

where $\tau \equiv \mathrm{i} t, t$ being the time

$$
\frac{\mathrm{d} \hat{a}_{\boldsymbol{k} \sigma}^{+}(\tau)}{\mathrm{d} \tau}=\left[\hat{H}, \hat{a}_{\boldsymbol{k} \sigma}^{+}(\tau)\right] .
$$

In SFA, we assume that the total Hamiltonian is given as a linear combination of the local-field operator $\hat{E}_{\boldsymbol{k} \sigma}$ and the number-of-particles operator $\hat{n}_{\boldsymbol{k} \sigma}=\hat{a}_{\boldsymbol{k} \sigma}^{+} \hat{a}_{\boldsymbol{k} \sigma}$ [21]:

$$
\hat{H}=\sum_{\boldsymbol{k} \sigma} \hat{E}_{\boldsymbol{k} \sigma} \hat{a}_{\boldsymbol{k} \sigma}^{+} \hat{a}_{\boldsymbol{k} \sigma}
$$

the indices $\boldsymbol{k}$ and $\sigma$ corresponding to given wavevectors and spin projections, respectively.

For fermions, the operator algebra for the creation and annihilation operators is defined by the following three anti-commutation relations:

$$
\begin{aligned}
& \left\{\hat{a}_{\boldsymbol{k} \sigma}, \hat{a}_{\boldsymbol{q} \lambda}^{+}\right\}=\delta_{\boldsymbol{k} \boldsymbol{q}} \delta_{\sigma \lambda} ; \quad\left\{\hat{a}_{\boldsymbol{k} \sigma}^{+}, \hat{a}_{\boldsymbol{q} \lambda}^{+}\right\}=0, \\
& \left\{\hat{a}_{\boldsymbol{k} \sigma}, \hat{a}_{\boldsymbol{q} \lambda}\right\}=0 .
\end{aligned}
$$

Assuming that the local-field operator $\hat{E}_{\boldsymbol{k} \sigma}$ is Hermitian and that it commutes with creation and annihilation operators, one obtains from Eq. (10):

$$
\frac{\mathrm{d} \hat{a}_{\boldsymbol{k} \sigma}^{+}(\tau)}{\mathrm{d} \tau}=\left[\hat{H}, \hat{a}_{\boldsymbol{k} \sigma}^{+}(\tau)\right]=\hat{E}_{\boldsymbol{k} \sigma}(\tau) \hat{a}_{\boldsymbol{k} \sigma}^{+}(\tau) .
$$

In SFA, we assume that the square of the quadratic fluctuation operator is replaced with its mean value

$$
\left(\Delta \hat{E}_{\boldsymbol{k}}\right)^{2} \cong\left\langle\left(\Delta \hat{E}_{\boldsymbol{k}}\right)^{2}\right\rangle
$$

where $\Delta \hat{E}_{\boldsymbol{k}} \equiv \hat{E}_{\boldsymbol{k}}-\left\langle\hat{E}_{\boldsymbol{k}}\right\rangle$ is the corresponding fluctuation of the mean-field operator from its mean value. It follows that the local-field fluctuation operator has two symmetric eigenvalues:

$$
\left(\Delta \hat{E}_{\boldsymbol{k}}\right)^{2} \cong\left\langle\left(\Delta \hat{E}_{\boldsymbol{k}}\right)^{2}\right\rangle=\varphi_{k}^{2}
$$

This is the single key assumption in this approximation. The implication is that not only the mean value of the spectrum is taken into account, but also the fluctuations of the spectrum around the mean value of the local-field operator [21].

Following a procedure closely related to that used for liquid ${ }^{3} \mathrm{He}[22]$, we obtain the final form of the long-range equation

$$
\left\langle\hat{n}_{\boldsymbol{k}} \hat{A}\right\rangle=\eta_{0}(k)\langle\hat{A}\rangle+\eta_{1}(k)\left\langle\Delta \hat{E}_{\boldsymbol{k}} \hat{A}\right\rangle,
$$

where $\hat{A}$ is an arbitrary operator that commutes with creation and annihilation operators. The functions $\eta_{0}(k)$ and $\eta_{1}(k)$ are given by

$$
\begin{gathered}
\eta_{0}(k) \equiv \frac{1}{2}\left[\frac{1}{1+\exp \left(\beta\left(\left\langle\hat{E}_{k}\right\rangle+\varphi_{k}\right)\right)}\right. \\
\left.+\frac{1}{1+\exp \left(\beta\left(\left\langle\hat{E}_{k}\right\rangle-\varphi_{k}\right)\right)}\right], \\
\eta_{1}(k) \equiv \frac{1}{2 \varphi_{k}}\left[\frac{1}{1+\exp \left(\beta\left(\left\langle\hat{E}_{k}\right\rangle+\varphi_{k}\right)\right)}\right. \\
\left.-\frac{1}{1+\exp \left(\beta\left(\left\langle\hat{E}_{k}\right\rangle-\varphi_{k}\right)\right)}\right] .
\end{gathered}
$$

$\beta \equiv\left(k_{\mathrm{B}} T\right)^{-1}$ is the inverse thermodynamic temperature, $k_{\mathrm{B}}$ being Boltzmann's constant, and $T$ the absolute temperature.

\subsubsection{Local field operator}

In treating the electron fluid at finite temperatures, it is convenient to use the grand canonical ensemble, which allows for the possibility of a variable number of parti- 
cles. The grand canonical Hamiltonian may be written in second quantization as

$$
\begin{aligned}
\hat{H} & =\sum_{\boldsymbol{k} \sigma}\left(\frac{\hbar^{2} k^{2}}{2 m}-\mu\right) \hat{a}_{\boldsymbol{k} \sigma}^{+} a_{\boldsymbol{k} \sigma} \\
& +\frac{1}{2 \Omega} \sum_{\sigma \lambda} \sum_{\boldsymbol{k} \boldsymbol{p}, \boldsymbol{q} \neq 0} V(q) \hat{a}_{\boldsymbol{k}+\boldsymbol{q}, \sigma}^{+} \hat{a}_{\boldsymbol{p}-\boldsymbol{q}, \lambda}^{+} a_{\boldsymbol{p} \lambda} a_{\boldsymbol{k} \sigma},
\end{aligned}
$$

$\mu$ being the chemical potential, defined as the energy required to remove (add) a particle from (to) the system at constant volume.

Using (12) and (18) for the local-field operator $\hat{E}_{\boldsymbol{k}}$, we find that

$$
\begin{aligned}
& \hat{E}_{\boldsymbol{k} \sigma}=\left\{\hat{a}_{\boldsymbol{k} \sigma},\left[\hat{H}, \hat{a}_{\boldsymbol{k}}^{+}\right]\right\}, \\
& {\left[\hat{H}, \hat{a}_{\boldsymbol{k} \sigma}^{+}\right]=\varepsilon_{\boldsymbol{k} \sigma} \hat{a}_{\boldsymbol{k} \sigma}^{+}+\frac{1}{2 \Omega} \sum_{\substack{\boldsymbol{k} \boldsymbol{p}, \boldsymbol{q} \neq 0 \\
\sigma \lambda}} V(q)} \\
& \quad \times\left(\hat{a}_{\boldsymbol{k}+\boldsymbol{q} \sigma}^{+} \hat{a}_{\boldsymbol{p}-\boldsymbol{q} \lambda}^{+} \hat{a}_{\boldsymbol{p} \lambda}-\hat{a}_{\boldsymbol{k}+\boldsymbol{q} \sigma}^{+} \hat{a}_{\boldsymbol{k}-\boldsymbol{q} \sigma}^{+} \hat{a}_{\boldsymbol{k} \sigma}\right) .
\end{aligned}
$$

The local-field operator $\hat{E}_{\boldsymbol{k}}$ is then given as

$$
\begin{aligned}
\hat{E}_{\boldsymbol{k} \sigma} & =\left\{\hat{a}_{\boldsymbol{k} \sigma},\left[\hat{H}, \hat{a}_{\boldsymbol{k} \sigma}^{+}\right]\right\} \\
= & \varepsilon_{\boldsymbol{k} \sigma}(\boldsymbol{k})-\frac{1}{\Omega} \sum_{\boldsymbol{p} \neq \boldsymbol{k}} V(|\boldsymbol{p}-\boldsymbol{k}|) \hat{n}_{\boldsymbol{p} \lambda} .
\end{aligned}
$$

Equation (21) makes clear that the local-field operator $\hat{E}_{\boldsymbol{k}}$ is spin-independent, which allows us to omit the spin indices. This reflects the fact that the interaction potential $V\left(\left|\boldsymbol{r}_{1}-\boldsymbol{r}_{2}\right|\right)$ is spin-independent; so is the particle distribution operator.

The corresponding mean value of the local-field operator is

$$
\left\langle\hat{E}_{\boldsymbol{k}}\right\rangle=\varepsilon_{k}(\boldsymbol{k})-\frac{1}{\Omega} \sum_{\boldsymbol{p} \neq \boldsymbol{k}} V(|\boldsymbol{p}-\boldsymbol{k}|)\left\langle\hat{n}_{\boldsymbol{p}}\right\rangle,
$$

where

$$
V(|\boldsymbol{p}-\boldsymbol{k}|)=4 \pi e^{2} /\left(q_{\mathrm{TF}}^{2}+|\boldsymbol{p}-\boldsymbol{k}|^{2}\right) .
$$

\subsubsection{Closed system of nonlinear integral equations}

Taking into account the symmetric property of the uniform electron fluid, $\left\langle\Delta \hat{E}_{\boldsymbol{k}}\right\rangle=0$, and assuming that in Eq. (17) $\hat{A}=1$, one gets

$$
\left\langle\hat{n}_{\boldsymbol{k}}\right\rangle=\eta_{0}(k) \text {. }
$$

In the equilibrium state, the electrons prefer energetically to stay in the lowest-momentum state; so if the fluctuations in the local-field operator lead to an increase in the state energy, then the fluctuations in the number of particles lead to a decrease in the number of particles in this state. This implies that the fluctuations in the number-of-particles operator are given by

$$
\hat{n}_{\boldsymbol{k}} \equiv\left\langle\hat{n}_{\boldsymbol{k}}\right\rangle-\Delta \hat{n}_{\boldsymbol{k}} \text {. }
$$

Rewriting the long-range equation in terms of the fluc- tuations of the occupation-number operator, we have

$$
\left\langle\Delta \hat{n}_{\boldsymbol{k}} \hat{A}\right\rangle=-\eta_{1}(k)\left\langle\Delta \hat{E}_{\boldsymbol{k}} \hat{A}\right\rangle \text {. }
$$

It is possible to obtain a closed set of nonlinear integral equations for the pair-correlation function $\left\langle\Delta \hat{n}_{\boldsymbol{k}} \Delta \hat{n}_{\boldsymbol{q}}\right\rangle_{c}$, the index $c$ denoting the true correlations for $\boldsymbol{k} \neq \boldsymbol{q}$ : substituting $\hat{A}$ by $\Delta \hat{n}_{\boldsymbol{q}}$ in (26), we get

$$
\begin{aligned}
& \left\langle\Delta \hat{n}_{\boldsymbol{k}} \Delta \hat{n}_{\boldsymbol{q}}\right\rangle_{c}=-\eta_{1}(k)\left\langle\Delta \hat{E}_{\boldsymbol{k}} \Delta \hat{n}_{\boldsymbol{q}}\right\rangle_{c} \\
& =\frac{\eta_{1}(k)}{\Omega} \sum_{\boldsymbol{p} \neq \boldsymbol{k}} V(|\boldsymbol{p}-\boldsymbol{k}|)\left\langle\Delta \hat{n}_{\boldsymbol{p}} \Delta \hat{n}_{\boldsymbol{q}}\right\rangle .
\end{aligned}
$$

The correlation function $\left\langle\Delta \hat{n}_{\boldsymbol{p}} \Delta \hat{n}_{\boldsymbol{q}}\right\rangle$ can then be written as

$$
\begin{aligned}
& \left\langle\Delta \hat{n}_{\boldsymbol{p}} \Delta \hat{n}_{\boldsymbol{q}}\right\rangle=\left\langle\left(\Delta \hat{n}_{\boldsymbol{q}}\right)^{2}\right\rangle \delta_{\boldsymbol{p q}}+\left\langle\Delta \hat{n}_{\boldsymbol{p}} \Delta \hat{n}_{\boldsymbol{q}}\right\rangle_{c}, \\
& \left\langle\left(\Delta \hat{n}_{\boldsymbol{q}}\right)^{2}\right\rangle \equiv\left\langle\hat{n}_{\boldsymbol{q}}^{2}\right\rangle-\left\langle\hat{n}_{\boldsymbol{q}}\right\rangle^{2} .
\end{aligned}
$$

For Fermi systems, the Pauli exclusion principle implies that $\hat{n}_{\boldsymbol{q}}^{2}=\hat{n}_{\boldsymbol{q}}$; so that (29) becomes

$$
\left\langle\left(\Delta \hat{n}_{\boldsymbol{q}}\right)^{2}\right\rangle=\left\langle\hat{n}_{\boldsymbol{q}}\right\rangle\left(1-\left\langle\hat{n}_{\boldsymbol{q}}\right\rangle\right) .
$$

Putting $\hat{A}=\Delta \hat{E}_{\boldsymbol{k}}$ in (26), we have

$$
\eta_{1}(k) \varphi_{k}^{2}=\frac{1}{\Omega} \sum_{\boldsymbol{p} \neq \boldsymbol{k}} V(|\boldsymbol{p}-\boldsymbol{k}|)\left\langle\Delta \hat{n}_{\boldsymbol{p}} \Delta \hat{n}_{\boldsymbol{k}}\right\rangle .
$$

The set of nonlinear integral Eqs. (16), (22), (27), (29) and (31) can be solved numerically by an iteration method using Gaussian quadrature for the static screened Coulomb potential.

\section{Calculations}

In the thermodynamic limit, and going over from the summation over momentum $\boldsymbol{p}$ to integration, we obtain from (22), (27) and (31)

$$
\begin{aligned}
& \left\langle\hat{E}_{k}\right\rangle=\varepsilon(k)-\int_{0}^{\infty} \mathrm{d} p p_{p \neq k}^{2} f(k, p)\left\langle\hat{n}_{p}\right\rangle \\
& \left\langle\Delta \hat{n}_{k} \Delta \hat{n}_{q}\right\rangle_{c}=\eta_{1}(k) \int_{0}^{\infty} \mathrm{d} p p_{p \neq k}^{2} f(k, p)\left\langle\Delta \hat{n}_{p} \Delta \hat{n}_{q}\right\rangle \\
& \eta_{1}(k) \varphi_{k}^{2}=\int_{0}^{\infty} \mathrm{d} p p_{p \neq k}^{2} f(k, p)\left\langle\Delta \hat{n}_{p} \Delta \hat{n}_{k}\right\rangle \\
& f(k, p) \equiv \frac{1}{(2 \pi)^{3}} \int V(|\boldsymbol{p}-\boldsymbol{k}|) \mathrm{d} \Theta
\end{aligned}
$$

where the integral of $V(|\boldsymbol{p}-\boldsymbol{k}|)$ is over the solid angle $\Theta$.

The mean value of the local-energy operator is given in its final form as

$$
\begin{gathered}
\left\langle\hat{E}_{k}\right\rangle=\varepsilon(k)-\int_{0}^{\infty} \mathrm{d} p p^{2}\left(\frac{e^{2}}{2 \pi k p}\right) \\
\times \ln \left[\frac{q_{\mathrm{TF}}^{2}+(p+k)^{2}}{q_{\mathrm{TF}}^{2}+(p-k)^{2}}\right]\left\langle\hat{n}_{p}\right\rangle .
\end{gathered}
$$


Here and throughout, we have used the natural system of units in which $\hbar=1, m=1$. Thus, the energy is given in $\AA^{-2}$.

This set of nonlinear integral equations can be used straightforwardly to obtain the physical characteristics of the system; in particular, the thermodynamics and pair-correlation function.

\subsection{Thermodynamic properties}

The knowledge of the grand partition function $Q$ allows us to determine all thermodynamic properties

$$
\ln Q=\sum_{\boldsymbol{p} \sigma} q_{0}(p),
$$

where

$$
\begin{gathered}
q_{0}(p) \equiv \frac{1}{2}\left[\ln \left(1+\exp \left(-\beta\left(\left\langle\hat{E}_{p}\right\rangle+\varphi_{p}\right)\right)\right)\right. \\
\left.+\ln \left(1+\exp \left(-\beta\left(\left\langle\hat{E}_{p}\right\rangle-\varphi_{p}\right)\right)\right)\right] .
\end{gathered}
$$

The grand mean energy can be expressed in terms of $Q$ as

$$
\begin{aligned}
U_{\mathrm{g}} & =\langle\hat{H}\rangle=-\left(\frac{\partial \ln (Q)}{\partial \beta}\right) \\
& =\sum_{\boldsymbol{p}}\left[\left\langle\hat{E}_{\boldsymbol{p}}\right\rangle\left\langle\hat{n}_{\boldsymbol{p}}\right\rangle+\eta_{1}(p) \varphi_{p}^{2}\right] .
\end{aligned}
$$

The pressure is

$$
P=k_{\mathrm{B}} T \frac{\ln Q}{\Omega} .
$$

The entropy can be calculated in terms of the grand mean energy $U_{\mathrm{g}}=U-\mu N$ and $P$ :

$$
S=\frac{1}{T}\left(U_{\mathrm{g}}+P \Omega\right) .
$$

Finally, the heat capacity of the system at constant volume is

$$
C_{v}=\left(\frac{\partial U_{\mathrm{g}}}{\partial T}\right)_{\Omega} .
$$

\subsection{Pair-correlation function}

The pair-correlation function $g_{\sigma 1 \sigma 2}\left(\boldsymbol{r}_{1}, \boldsymbol{r}_{2}\right)$ is related to the probability of finding an electron of spin $\sigma_{1}$ at $\boldsymbol{r}_{1}$ and a second electron of spin $\sigma_{2}$ at $\boldsymbol{r}_{2}$. Since it is a probability, it must be positive everywhere: $g(\boldsymbol{r}) \geq 0$ [27-31]. The Pauli exclusion principle implies that $g_{\sigma \sigma}(0)=0$. Physically, $n_{\sigma 2}\left(\boldsymbol{r}_{2}\right) g_{\sigma 1 \sigma 2}\left(\boldsymbol{r}_{1}, \boldsymbol{r}_{2}\right) d^{3} \boldsymbol{r}_{2}$ is the expected number of electrons in the volume element $d^{3} \boldsymbol{r}_{2}$ at $\boldsymbol{r}_{2}$, given that there is an electron at position $\boldsymbol{r}_{1}$. In the unpolarized electron fluid, the electronic spin density $n_{\uparrow}(\boldsymbol{r})=$ $n_{\downarrow}(\boldsymbol{r})=n(\boldsymbol{r}) / 2$ is uniform in space; so that $g_{\sigma 1 \sigma 2}\left(\boldsymbol{r}_{1}, \boldsymbol{r}_{2}\right)$ depends only on the relative coordinates $\boldsymbol{r}=\left|\boldsymbol{r}_{1}-\boldsymbol{r}_{2}\right|$ between the two electrons.

For noninteracting electrons, the pair correlation function is given in the Hartree-Fock approximation $[10,27,32]$ by

$$
g_{\sigma 1 \sigma 2}(r)=1-9\left(\frac{j_{1}\left(k_{\mathrm{F}} r\right)}{k_{\mathrm{F}} r}\right)^{2} \delta_{\sigma 1 \sigma 2},
$$

$j_{1}(r)$ being the spherical Bessel function.

The more general form of the pair correlation function for interacting electrons is expressed as [31, 33, 34]

$$
\begin{aligned}
& n_{\sigma 1}\left(\boldsymbol{r}_{1}\right) n_{\sigma 2}\left(\boldsymbol{r}_{2}\right) g_{\sigma 1 \sigma 2}\left(\boldsymbol{r}_{1}, \boldsymbol{r}_{2}\right) \\
& \quad=\left\langle\Phi\left|\hat{\psi}_{\sigma 1}^{+}\left(\boldsymbol{r}_{1}\right) \hat{\psi}_{\sigma 2}^{+}\left(\boldsymbol{r}_{2}\right) \hat{\psi}_{\sigma 2}\left(\boldsymbol{r}_{2}\right) \hat{\psi}_{\sigma 1}\left(\boldsymbol{r}_{1}\right)\right| \Phi\right\rangle,
\end{aligned}
$$

where $n_{\sigma}(\boldsymbol{r})$ is the electron density operator for the spin projection $\sigma=\uparrow$ or $\downarrow$, and the expectation value is taken over $\Phi$, the ground state of the system. This definition ensures the symmetry property $g_{\sigma 1 \sigma 2}\left(\boldsymbol{r}_{1}, \boldsymbol{r}_{2}\right)=$ $g_{\sigma 2 \sigma 1}\left(\boldsymbol{r}_{2}, \boldsymbol{r}_{1}\right)$, and the asymptotic value $g_{\sigma 1 \sigma 2}(\boldsymbol{r} \rightarrow \infty)=$ 1 , denoting no correlations between two electrons in this limit.

In second quantization,

$$
\begin{aligned}
& g_{\sigma 1 \sigma 2}\left(\boldsymbol{r}_{1}, \boldsymbol{r}_{2}\right)=\frac{1}{4 n^{2} \Omega^{2}} \sum_{\boldsymbol{k}, \boldsymbol{p}}\left[\left\langle\hat{n}_{\boldsymbol{k}, \sigma 1} \hat{n}_{\boldsymbol{p}, \sigma 2}\right\rangle\right. \\
& \left.-\left\langle\hat{n}_{\boldsymbol{k}, \sigma 1} \hat{n}_{\boldsymbol{p}, \sigma 1}\right\rangle \mathrm{e}^{-\mathrm{i}(\boldsymbol{k}-\boldsymbol{p}) \cdot \boldsymbol{r}}\right] .
\end{aligned}
$$

Changing the summation over $\boldsymbol{k}$ and $\boldsymbol{p}$ to integrals, one can express the spin parallel and spin anti-parallel pair-correlation functions within SFA theory as

$$
\begin{aligned}
& g_{\uparrow \uparrow}(\boldsymbol{r})=\frac{1}{\pi^{4} n^{2}} \int_{0}^{\infty} \mathrm{d} k k^{2} \int_{0}^{\infty} \mathrm{d} p p^{2}\left(1-j_{0}(k r) j_{0}(p r)\right) \\
& \times\left[\left\langle\Delta \hat{n}_{\boldsymbol{k} \uparrow} \Delta \hat{n}_{\boldsymbol{p} \uparrow}\right\rangle_{\boldsymbol{k} \neq \boldsymbol{p}}+\left\langle\hat{n}_{\boldsymbol{k} \uparrow}\right\rangle\left\langle\hat{n}_{\boldsymbol{p} \uparrow}\right\rangle\right],
\end{aligned}
$$

and

$$
\begin{aligned}
& g_{\uparrow \downarrow}(\boldsymbol{r})=\frac{1}{\pi^{4} n^{2}} \int_{0}^{\infty} \mathrm{d} k k^{2} \int_{0}^{\infty} \mathrm{d} p p^{2}\left[\left\langle\Delta \hat{n}_{\boldsymbol{k} \uparrow} \Delta \hat{n}_{\boldsymbol{p} \downarrow}\right\rangle\right. \\
& \left.\quad+\left\langle\hat{n}_{\boldsymbol{k} \uparrow}\right\rangle\left\langle\hat{n}_{\boldsymbol{p} \downarrow}\right\rangle\right] .
\end{aligned}
$$

\section{Results and discussion}

In this section we summarize the results obtained within the SFA framework for the thermodynamic properties as well as for the pair-correlation function of the electron fluid, in which the density parameter $r_{\mathrm{s}}$ is less than the melting point of the Wigner crystal. The present approach is free of any input and/or fitting parameters.

Let us first explore the screening effect on the mean energy per particle $(\varepsilon \equiv U / N)$ by choosing different values for the screening parameter ratio: $\kappa=0.0 q_{\mathrm{TF}}$ (the bare interaction); $\kappa=0.3 q_{\mathrm{TF}} ; \kappa=0.5 q_{\mathrm{TF}} ;$ and $\kappa=1.0 q_{\mathrm{TF}}$ (the full interaction); i.e., $\kappa$ ranges from 0 for a non-screened potential to $q_{\mathrm{TF}}$ for a fully-screened potential.

Figure 1 displays schematically our $\varepsilon$ results for various $\kappa$ in terms of $r_{\mathrm{s}}$ at fixed temperature, $T=1000 \mathrm{~K}$. $\varepsilon$ has a "standard" shape consisting of a repulsive barrier at short range followed by an attractive well, as indicated by Eq. (22). For all $\kappa$-values, the screened Coulomb energy is somewhat higher than that for the 
corresponding bare Coulomb energy. Furthermore, the energies converge rapidly to a limiting value when $r_{\mathrm{s}}$ increases. The minimum of the bare Coulomb potential occurs at $r_{\mathrm{s}}=2.6$ with $\varepsilon_{\min }=-0.576 \AA^{-2}(-4.389 \mathrm{eV})$; while the minimum of the full-screened potential occurs at $r_{\mathrm{s}}=4.0$, which is identical to that of Ref. [35], with $\varepsilon_{\min }=-0.119 \AA^{-2}(-0.909 \mathrm{eV})$. This means that the location of the energy minimum depends on $r_{\mathrm{s}}$ (i.e., density). It is interesting to observe that the present values compare favorably to the experimental values for metallic sodium, in which $r_{\mathrm{s}}=3.96$ and $\varepsilon_{\min }=-1.13 \mathrm{eV}$ [1]. The negative values of the $\varepsilon$ minima indicate that the system is bound. These minima shift upwards and slightly towards higher $r_{\mathrm{s}}$ values as $\kappa$ increases.

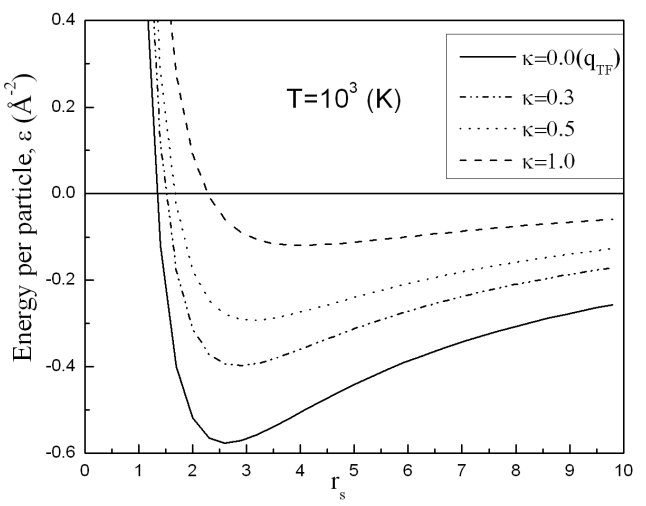

Fig. 1. The energy per particle, $\varepsilon$, for different values of the screening parameter ratio $\kappa$, as a function of the dimensionless parameter $r_{\mathrm{s}}$. The solid curve is for the bare Coulomb interaction; the dashed-dotted and dotted curves are for partial screening; and the dashed curve is for full screening.

At lower $\kappa, \varepsilon$ deepens, which means that the screening effect reduces the interparticle potential, as expected physically.

The kinetic energy dominates in the high-density region, where the system behaves, therefore, like an ideal electron gas. It is well known that for a fermion system at high density $\varepsilon$ goes as $r_{\mathrm{s}}^{-2}$ at temperatures below the Fermi temperature $T_{\mathrm{F}}$ [1]. Thus, in this density region, both the screened and the bare Coulomb interactions do not have a major effect on $\varepsilon$. The electron fluid has the peculiar property of becoming more and more ideal as its density rises.

In Fig. 2, $\varepsilon$ is shown as a function of $r_{\mathrm{s}}$ for the electron fluid. The results of this work, with the full static screened potential $\left(\kappa=q_{\mathrm{TF}}\right)$, will now be compared to those of other various theories, which include certain approximations. Clearly, our results are in excellent agreement with more complicated many-body theories. In fact, there is no noticeable difference between the present calculations and those of Ref. [36] over the whole range of $r_{\mathrm{s}}$ as well as those of Refs. [37, 38] within $r_{\mathrm{s}} \leq 5$, which correspond to typical metallic densities. This is to be expected, since the results of Refs. [36-38] are calculated at high density. For $r_{\mathrm{s}} \geq 5$, we get a similar density-dependence behavior to that of Refs. [4, 13, 14], where the variational Monte Carlo and fixed-node diffusion Monte Carlo techniques were used, and of Ref. [15], where the Fermi hypernetted-chain theory was used. Further, our energy results for full screening are virtually identical to the corresponding empirical results of Refs. [35-39] in the high-density region.

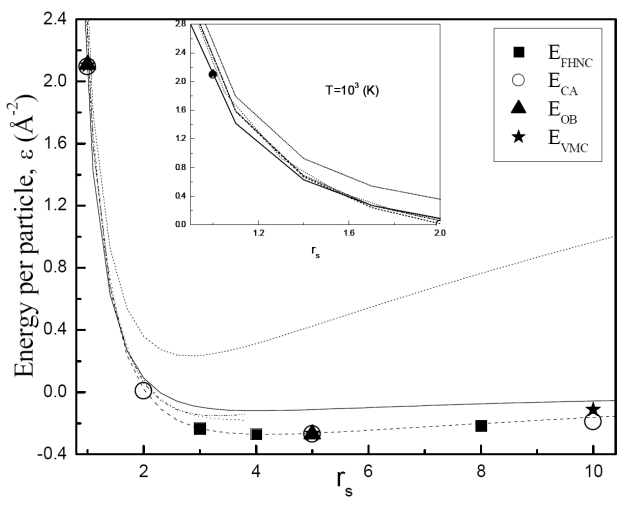

Fig. 2. The energy per particle, $\varepsilon$, as a function of $r_{\mathrm{s}}$. The solid curve shows the results obtained in this work for full screening. The dotted curve represents the results of Ref. [36]; the dashed line refers to the calculations of Ref. [40]; the dashed-dotted curve is that of Refs. [37, 38]; and the short dashed curve is that of Ref. [39]. The FHNC-values correspond to the Fermi hypernetted-chain theory results, Ref. [15]; CA-values refer to Ceperley and Alder, Ref. [5]; OB-values to Ortiz and Ballone, Refs. [13, 41]; finally, VMC-values to Kwon et al., Ref. [14]. The behavior of the energy per particle, $\varepsilon$, at small $r_{\mathrm{s}}(0-2)$ is shown in the inset.

In Figs. 3-7, the thermodynamic properties for the same $\kappa$-values are shown as functions of the density parameter $r_{\mathrm{s}}$ at $T=1000 \mathrm{~K}$. Both pressure and entropy fall rapidly to zero with increasing $r_{\mathrm{s}}$. When $r_{\mathrm{s}}$ increases further, particularly at $r_{\mathrm{s}} \geq 2$, the pressure and the entropy curves become identical for both bare and screened Coulomb potentials. The degree of order of the system decreases as $\kappa$ increases at the same density, as shown in Fig. 4. In contrast to the entropy, however, there is a monotonic decrease in the pressure when the screening effect is included, as shown in Figs. 3, 4. On the other hand, at low density, the concentration of the particles in the system is less than at high density, which reduces both the pressure and the entropy in this latter density range.

Other thermodynamic properties that have been studied are the chemical potential $\mu$, the Helmholtz free energy $F$, and the heat capacity $C_{v}$. These are displayed in Figs. 5, 6 and 7, respectively. With increasing $r_{\mathrm{s}}, \mu$ as well as $C_{v}$ decrease steadily, whereas $F$ increases.

The chemical potential is calculated from the condition $N=\sum_{\boldsymbol{k}}\left\langle\hat{n}_{\boldsymbol{k}}\right\rangle$. As shown in Fig. 5, for full screening $\mu$ decreases steadily with increasing $r_{\mathrm{s}}$, but remains positive. In this case, the system behaves like an ideal Fermi gas 


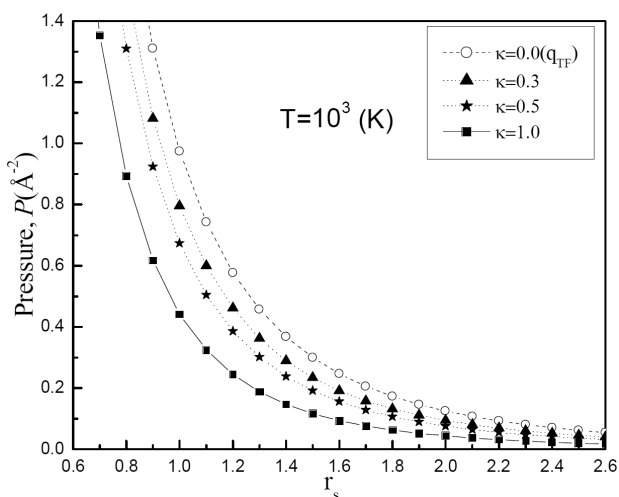

Fig. 3. The $r_{\mathrm{s}^{-}}$(i.e., density-) dependence of the pressure for the electron fluid with the static screened potential for various values of $\kappa$. This is essentially the equation of state for the system.

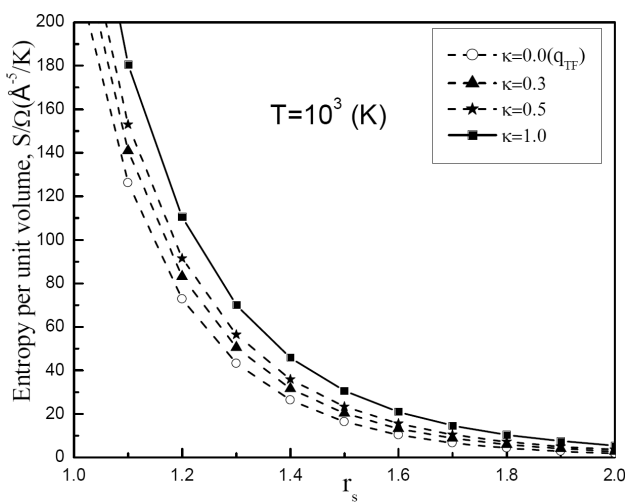

Fig. 4. The entropy per unit volume, $S / \Omega$, for the electron fluid as a function of $r_{\mathrm{s}}$ for different values of $\kappa$.

in its ground state $\left(\mu \propto r_{\mathrm{s}}^{-2}\right)$. This behavior is readily understood, the lowest energy state of the system being obtained by filling the energy levels up to $\mu=\varepsilon_{\mathrm{F}}$. Clearly, there is a tangible change in the behavior of $\mu$ with decreasing $r_{\mathrm{s}}$ at different $\kappa$. For $r_{\mathrm{s}} \leq 5, \mu$ is highly-dependent on density, but becomes almost constant at $r_{\mathrm{s}} \geq 5$. This is because, in the high-density region, the system needs much more energy to add (remove) a particle. For the bare Coulomb potential, where $\mu$ is negative, the system behaves just like the classical ideal gas; while for full screening, where $\mu$ is positive definite, the system is quantal. It is recalled in this connection that in the classical ideal gas (the Boltzmann statistics) $\mu$ always takes negative values (of large absolute value); whereas in the Fermi statistics $\mu$ can be positive or negative. This underlines the profound effect of the screening effect on $\mu$.

The free-energy curve, shown in Fig. 6, has a linear behavior for small $r_{\mathrm{s}}$, where the system behaves like an ideal gas. As $r_{\mathrm{s}}$ increases, $F$ for both bare and screened Coulomb potentials increase steadily to a limiting value. Clearly, screening has a maximal effect on $F$ at all temperatures considered.

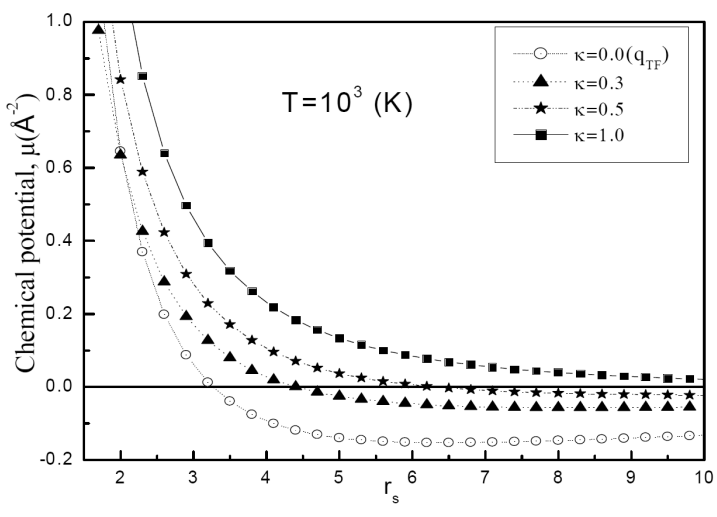

Fig. 5. The chemical potential $\mu$ versus $r_{\mathrm{s}}$ for different $\kappa$-values at fixed temperature $(T=1000 \mathrm{~K})$.

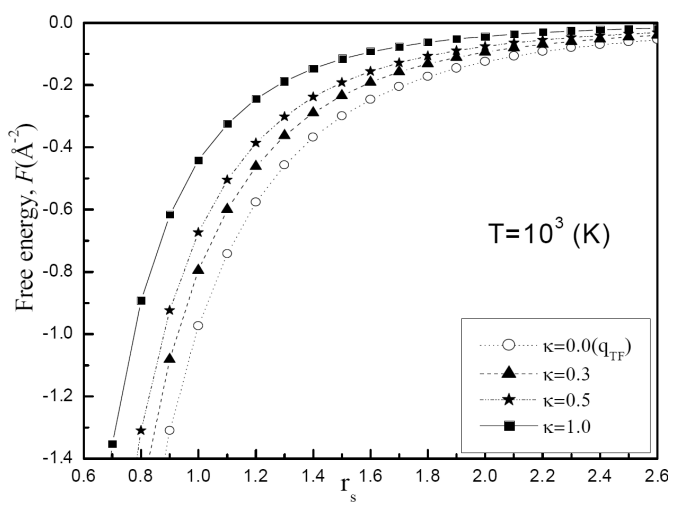

Fig. 6. The free energy, $F$, for the electron fluid, with a screened potential, versus $r_{\mathrm{s}}$ for selected values of $\kappa$.

The modification of the thermodynamic calculations by virtue of the screening effect is, however, well-reflected in $C_{v}$. Clearly, $C_{v}$ is more sensitive to $r_{\mathrm{s}}$ for the bare Coulomb potential than for the full-screened interaction, as shown in Fig. 7. In particular, a striking difference can be seen between the two potentials in the high-density

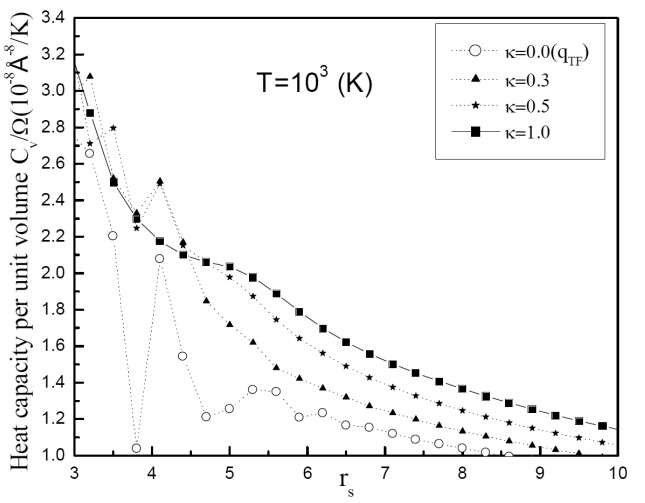

Fig. 7. The heat capacity per unit volume, $C_{v} / \Omega$, at $T=1000 \mathrm{~K}$ as a function of $r_{\mathrm{s}}$ for selected values of $\kappa$. 
region, unlike the low-density region. The oscillations in $C_{v}$ at low $r_{\mathrm{s}}$ may be spurious, but the general trend of the curves at different values of $\kappa$ could be real.

We turn now to the $T$-dependence of the thermodynamic properties. Our calculations were carried out for a fixed $r_{\mathrm{s}}=3.93$ (pertaining to $\mathrm{Na}$ ) in the $\mathrm{T}$-range $(1-9) \times 10^{4} \mathrm{~K}$. The resulting thermodynamic properties are plotted at various $T$ in Figs. 8-12.

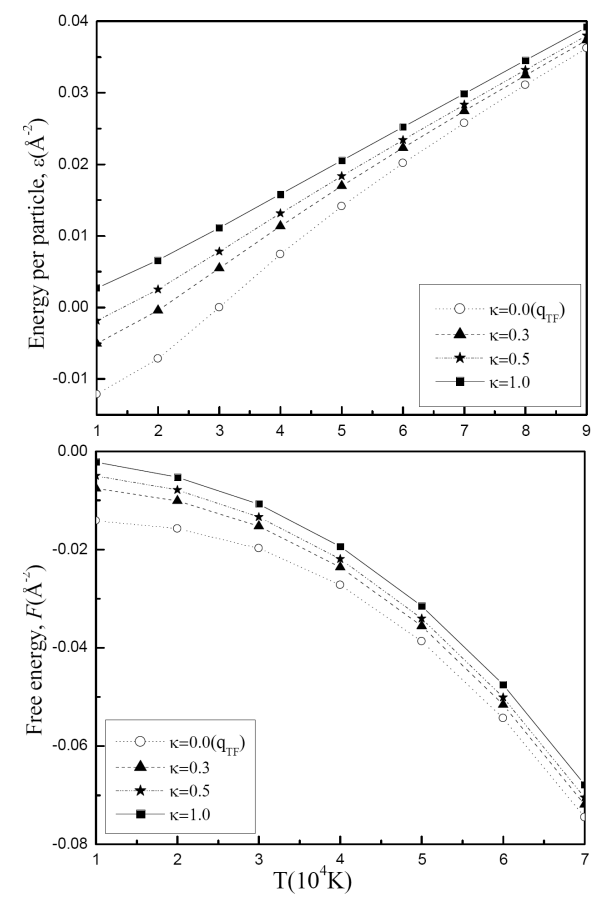

Fig. 8. The temperature-dependence of the energy per particle $\varepsilon$ (upper diagram), and the free energy $F$ (lower diagram) of the electron fluid at fixed $r_{\mathrm{s}}=3.93$ for different $\kappa$

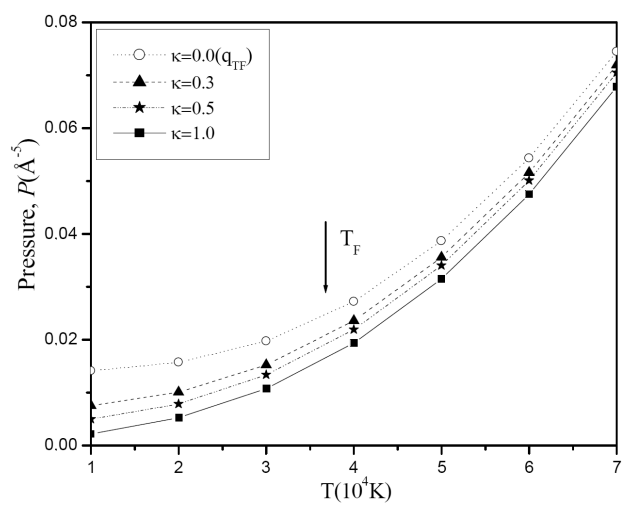

Fig. 9. The temperature-dependence of the pressure $P$ at fixed $r_{\mathrm{s}}=3.93$.

Clearly, there is a monotonic increase with $T$ of all quantities, except $\mu$ which decreases with $T$. As shown in Figs. 8-12, the screening effect "improves" the results of this work for $\varepsilon, F, S / \Omega$ and $\mu$.

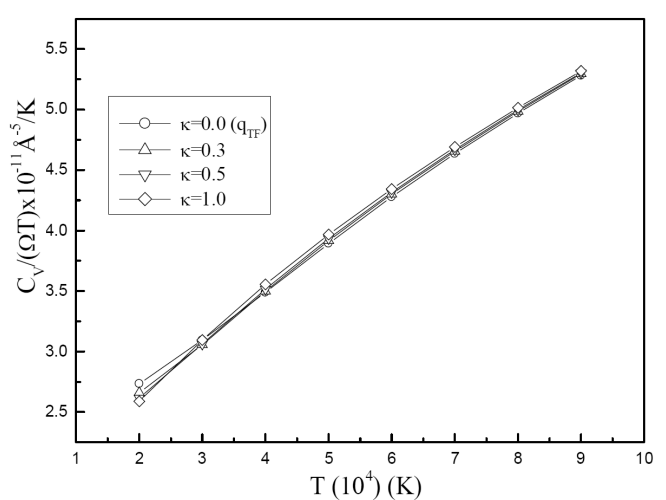

Fig. 10. The temperature-dependence of the heat capacity $C_{v} /(T \Omega)$ at fixed $r_{\mathrm{s}}=3.93$.

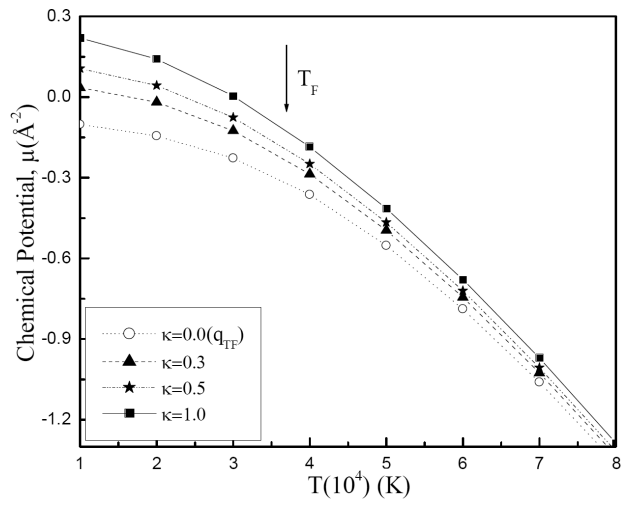

Fig. 11. The temperature-dependence of the chemical potential $\mu$ at fixed $r_{\mathrm{s}}=3.93$.

The energy per particle $\varepsilon$ (upper diagram) and the free energy $F$ (lower diagram) are plotted in Fig. 8 for different values of $\kappa$. The screening effect shifts $\varepsilon$ as well as $F$ to higher values, where the system is in scattering rather than bound states. For such density and low- $T$ ranges, $\varepsilon$ is almost proportional to $T$; but $F$ decreases rapidly to

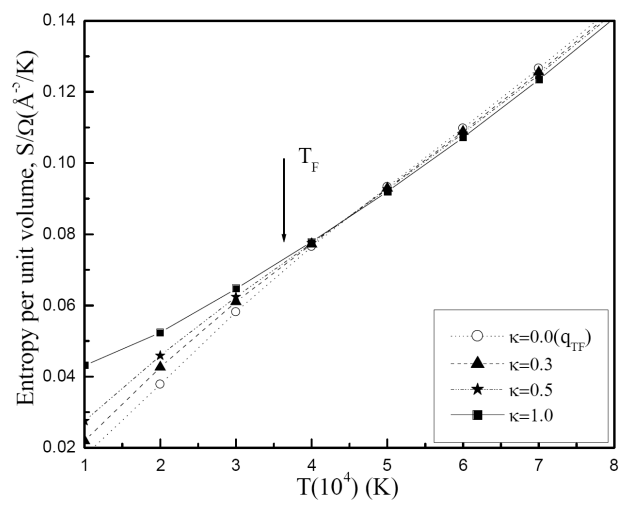

Fig. 12. The temperature dependence of the entropy per unit volume $S / \Omega$ at fixed $r_{\mathrm{s}}=3.93$ for various $\kappa$. 
a limiting value. However, screening has almost no effect on $P$ and $C_{v}$ at all temperatures considered.

The pressure $P$, which is plotted in Fig. 9 as a function of $T$, has almost a fixed value below the Fermi temperature $T_{\mathrm{F}} \approx 3.77 \times 10^{4} \mathrm{~K}$; but increases rapidly to a limiting value above this value. Clearly, the screening effect is to shift $P$-values in the regime $T<T_{\mathrm{F}}$, where the system behaves like as an ideal gas; but it has a reduced effect above $T_{\mathrm{F}}$, where the interparticle interaction has a major role.

The electronic specific heat is a fundamental quantity in the theory of electrons in metals. For ideal Fermi systems, the electronic specific heat capacity is linear with temperature [42]. The fitted curves of Fig. 10, $C_{v} /(T \Omega)$, are found to satisfy the linear relation $C_{v} /(T \Omega)=2+$ $0.36 T \AA^{-5} \mathrm{~K}^{-1}$. At very low temperatures, our results are close to the ideal case. As the temperature increases, the interaction plays a more appreciable role. Our results in Fig. 10 show that the electronic specific heat capacity is independent of the screening strength.

As shown in Fig. 11, $\mu$ decreases steadily below $T_{\mathrm{F}}$; but falls rapidly with increasing $T$. It is clear from the figure that $\mu$ for the screened Coulomb potential is greater than for the bare potential. The chemical potential decreases from $\varepsilon_{\mathrm{F}}$ at $T=0$, to smaller and smaller values, until $\mu=0$ just below $T_{\mathrm{F}}$ at which even the single-particle ground state is unlikely to be occupied. After this point, $\mu$ becomes negative. As $T$ rises, the electron fluid eventually begins to mimic the classical behavior: $\mu$ decreases and becomes increasingly negative.

The entropy per unit volume $S / \Omega$ is displayed in Fig. 12. $S / \Omega$ increases linearly as $T$ rises, more microstates becoming available. When $T$ rises further, $S / \Omega$ converges to a limiting value for both screened and bare Coulomb potentials. In this case, screening has little effect on the entropy, and the system behaves like an ideal gas.

As a next step in the present work, the pair-correlation function $g(r)$ is calculated at $T=1000 \mathrm{~K}$ for the system within the SFA scheme. Figure 13 displays our calculations for the smoothed pair-correlation function at different $r_{\mathrm{s}}$ values. Unlike in most standard theories of the electron fluid, where $g(r)$ becomes negative at small distances in the metallic range which is a well-known unphysical feature of these approximations, $g(r)$ obtained in the present calculations is positive definite for all values of distance and density. This function should approach one when $r$ becomes larger than the interparticle separation. In our work, $g(r)$ approaches 0.99 in this limit, which is close to one. This small discrepancy may be attributed to the accuracy less than $100 \%$ of the computer code used here.

The behavior of the pair-correlation function should satisfy the cusp condition $g_{\sigma_{1} \sigma_{2}}(r) \leq 1$ [33]. Our results shown in Figs. 13 and 14 satisfy this condition.

There is a disagreement between our results for the average pair-correlation function $g\left(r \rightarrow 0 ; r_{\mathrm{s}}\right)$ and those of quantum Monte Carlo calculations [34]. In our results,

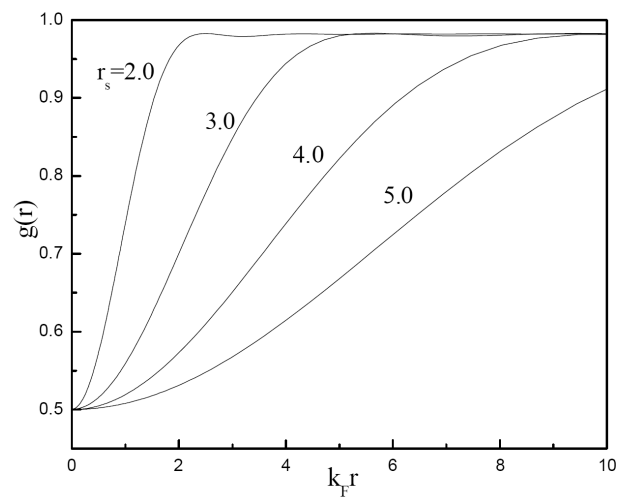

Fig. 13. The pair-correlation function $g(r)$ for the electron fluid as a function of $k_{\mathrm{F}} r$ for various densities.

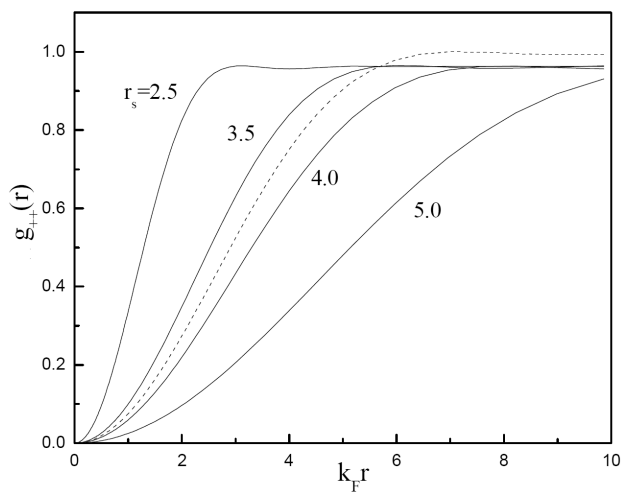

Fig. 14. The parallel pair-correlation function evaluated in the present approximation for various densities, compared to the Hartree-Fock result [10, 27, 28].

as $r \rightarrow 0$, this function $\rightarrow 0.5$; whereas in [34] $g_{\uparrow \downarrow}(r)$, goes to 0.25 as $r \rightarrow 0$, in the high-density limit and falls down to zero in the low-density limit. This means that the average pair-correlation function in [34] ranges from 0.25 at high density to zero at low density.

In spite of the discrepancy between our results and quantum Monte Carlo results as $r \rightarrow 0$, the general behavior of our pair-correlation function is consistent with those results, especially in the high-density limit. In quantum Monte Carlo calculations, $g_{\uparrow \downarrow}(r)$ increases drastically to its saturated value $(\approx 1)$, and the saturation point shifts up as the density decreases. In our work, the saturation point for the average pair-correlation function increases as the density decreases with a rate greater than in quantum Monte Carlo calculations [34].

In Fig. 14, our parallel pair-correlation function is plotted as a function of $k_{\mathrm{F}} r$ for various values of $r_{\mathrm{s}}$. For comparison purposes, the corresponding Hartree-Fock function is displayed in the same figure. This is repeated for the total pair-correlation function in Fig. 15. The two total pair-correlation functions have the same behavior.

The fundamental requirements for the parallel and total pair-correlation functions - evident from their defi- 


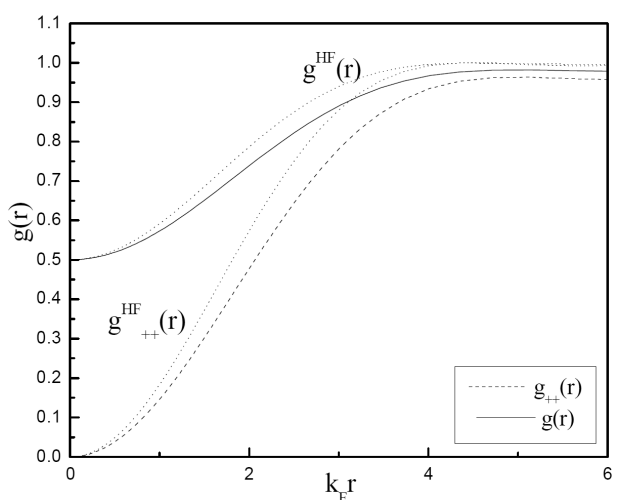

Fig. 15. The SFA-pair-correlation functions, $g(r)$ (solid curve) and $g_{\uparrow \uparrow}(r)$ (dashed line). The HartreeFock values, $g^{\mathrm{HF}}(r)$ and $g_{\uparrow \uparrow}^{\mathrm{HF}}(r)$, are also shown (dotted curves).

nitions, i.e., $g(r) \geq 0, g_{\uparrow \uparrow}(r) \geq 0$ and $g_{\uparrow \uparrow}(0)=0-$ are completely satisfied in the present calculations at any electron density.

In many published works, the cusp property $g_{\sigma_{1} \sigma_{2}}(r) \leq$ 1 is violated [27], and in other works, unphysical (negative) values of $g(r)$ are present [12, 43-45]. These unphysical features still plague many calculations of $g(r)$. On the other hand, in most previous papers, $g(r)$ reaches the saturation point at $\frac{r}{r_{\mathrm{s}}} \approx 1[27,33,34,46-48]$. In the present work, this behavior is missing; the saturation point depends on the system density.

It can easily be ascertained that the results of this work for the parallel and anti-parallel pair-correlation function, given by Eq. (47), are reduced to those in the HF approximation, given by Eq. (43), when the $\left\langle\Delta \hat{n}_{\boldsymbol{k}} \Delta \hat{n}_{\boldsymbol{p}}\right\rangle$ terms in the SFA scheme are ignored. This is reasonable because our work is, in effect, a generalized mean-field (HF) approximation.

The pair-correlation function at zero interparticle separation, $g(0)$, has been computed using SFA in the range $1 \leq r_{\mathrm{s}} \leq 6$. It is found that $g(0)=0.5$ over this range. In general, there are wide differences in the published values of the $g(0)[12,43-45,48-53]$. Gori-Giorgi et al. [34] found that $g(0)$ at high density, $r_{\mathrm{s}}=1$, is close to 0.25 , and decreases to zero at $r_{\mathrm{s}}=10$. Also, $g(r)$ was computed for the uniform electron gas using the Overhauser potential [50]. In the limit $r \rightarrow 0$, the results varied from 0.27 at $r_{\mathrm{s}}=1$ to zero at $r_{\mathrm{s}}=10$. The ladder theory was used by Zhixin [53] to calculate $g(0)$. From these and other calculations, it is evident that the dependence on the method used appears most sharply as the density decreases. Several other formalisms give negative values for $g(0)$ at sufficiently large $r_{\mathrm{s}}$ in the metallic range $[12,43-45,50-52]$. Since $g(0)$ must be positive, this unphysical feature should be given special attention. The inconsistency between our results and other published results may indicate that the Yukawa screening potential is not suitable at low densities.

\section{Conclusions}

In summary, we have presented in this work a theoretical study of the thermodynamic properties and of the pair-correlation function for the three-dimensional electron fluid. The calculations, based on the screening potential as the input interaction between electrons, have been undertaken with the static fluctuation approximation. Predictions of the present approach for the thermodynamic properties appear to be sound on general physical grounds and compare favorably to other many-body techniques. The best agreement is obtained in the weak- and intermediate-coupling strength. For the pair-correlation function, the present calculations give positive values for all densities. Furthermore, the pair-correlation at zero-interparticle separation is positive definite for any electron density. The results obtained are close to those given by the HF approximation, since our work is based on a generalized mean-field approximation.

\section{Acknowledgments}

One of us (H.B.G.) is grateful to the University of Jordan for granting him a sabbatical leave in the academic year 2010-2011, during which this work was completed under the general title "Many-Body Systems: Further Studies and Calculations [Part Two]".

\section{References}

[1] A.L. Fetter, J.D. Walecka, Quantum Theory of Many-Body Systems, McGraw-Hill, New York 1971.

[2] E.H. Lieb, J.L. Lebowitz, Adv. Math. 9, 316 (1972).

[3] G. Ortiz, M. Harris, P. Ballone, Phys. Rev. Lett. 82, 5317 (1999).

[4] E. Wigner, Phys. Rev. 46, 1002 (1934).

[5] D.M. Ceperley, B.J. Alder, Phys. Rev. Lett. 45, 566 (1980).

[6] D. Bohm, D. Pines, Phys. Rev. 82, 625 (1951).

[7] D. Pines, Phys. Rev. 92, 626 (1953).

[8] P. Nozières, D. Pines, Phys. Rev. 109, 741 (1958).

[9] O.V. Roos, Phys. Rev. 120, 1641 (1960).

[10] N.H. March, W.H. Young, S. Sampanthar, The Many-Body Problem in Quantum Mechanics, Dover Publication, New York 1995.

[11] U. von Barth, L. Hedin, J. Phys. C 5, 1629 (1972).

[12] K.S. Singwi, M.P. Tosi, R.H. Land, A. Sjölander, Phys. Rev. 176, 589 (1968).

[13] G. Ortiz, M. Harris, P. Ballone, Phys. Rev. B 50 , 1391 (1994).

[14] Y. Kwon, D.M. Ceperley, R.M. Martin, Phys. Rev. B 58, 6800 (1998).

[15] J.G. Zabolitzky, Phys. Rev. B 22, 2353 (1980).

[16] E. Krotscheck, W. Kohn, G.X. Qian, Phys. Rev. B 32, 5693 (1985).

[17] R.F. Bishop, K.H. Luhrmann, Phys. Rev. B 17, 3757 (1978). 
[18] R.F. Bishop, K.H. Luhrmann, Physica B 108, 873 (1981).

[19] P. Garcia-Gonzalez, R.W. Godby, Phys. Rev. B 63 , 075112 (2001).

[20] R.R. Nigmatullin, A.A. Khamzin, H.B. Ghassib, Phys. Rev. E 61, 3441 (2000).

[21] M.K. Al-Sugheir, H.B. Ghassib, R.R. Nigmatullin, Int. J. Theor. Phys. 40, 1033 (2001).

[22] M.K. Al-Sugheir, H.B. Ghassib, Int. J. Theor. Phys. 41, 705 (2002).

[23] B.R. Joudeh, M. Al-Sugheir, H.B. Ghassib, Int. J. Mod. Phys. B 19, 3985 (2005).

[24] N.M. Ghulam, H.B. Ghassib, M.K. Al- Sugheir, Phys. Rev. C 75, 064317 (2007).

[25] A. Alastuey, Ph. A. Martin, Europhys. Lett. 6, 385 (1988).

[26] A. Alastuey, Physica A 263, 271 (1999).

[27] Y. Ouasak, H. Suehiro, H. Yasuhara, J. Phys. C, Solid State Phys. 18, 4471 (1985).

[28] M.W.C. Dharma-Wardana, F. Perrot, Phys. Rev. Lett. 84, 959 (2000).

[29] F. Perrot, M.W.C. Dharma-Wardana, Phys. Rev. Lett. 87, 206404 (2001).

[30] F.H. Stillinger, S. Torquato, J. Phys. Chem. B 108, 19589 (2004).

[31] P.R. Antoniewicz, L. Kleiman, Phys. Rev. B 2, 2808 (1970).

[32] T.P. Fishlock, J.B. Pendry, J. Phys. C, Solid State Phys. 6, 1909 (1973).

[33] A.K. Rajagopal, J.C. Kimball, M. Banerjee, Phys. Rev. B 18, 2339 (1978).

[34] P.G. Giorgi, F. Sacchetti, G.B. Bachelet, Phys. Rev. B 61, 7353 (2000).

[35] J. Perdew, S. Kurth, Lecture Notes in Physics, Vol. 620, Springer, Berlin 2003, p. 1.
[36] M. Gell-Mann, K. Brueckner, Phys. Rev. 106, 364 (1957).

[37] W.J. Carr, Jr., A.A. Maradudin, Phys. Rev. 133, A371 (1964).

[38] T. Endo, M. Horiuchi, Y. Takada, H. Yasuhara, Phys. Rev. B 59, 7367 (1999).

[39] D.Y. Kojima, A. Isihara, Z. Phys. B 25, 167 (1976).

[40] V.C. Aguilera-Navarro, G.A. Baker, Jr., M. de Llano, Phys. Rev. B 32, 4502 (1985).

[41] G. Ortiz, P. Ballone, Europhys. Lett. 23, 7 (1993).

[42] N.W. Ashcroft, N.D. Mermin, Solid State Physics, Saunders College, Philadelphia 1976.

[43] K.S. Singwi, A. Sjölander, K.P. Tosi, R.H. Land, Solid State Commun. 7, 1503 (1969).

[44] K.N. Pathak, P. Vashishta, Phys. Rev. B 7, 3649 (1973).

[45] F. Toigo, T.O. Woodruff, Phys. Rev. B 4, 371 (1971).

[46] B. Davoudi, R. Asgari, M. Polini, M.P. Tosi, Phys. Rev. B 68, 155112 (2003).

[47] B. Davoudi, M. Polini, R. Asgari, M.P. Tosi, Phys. Rev. B 66, 075110 (2002).

[48] P. Gori-Giorgi, P.J. Perdew, Phys. Rev. B 64, 155102 (2001).

[49] L. Calmels, A. Gold, Phys. Rev. B 57, 1436 (1998).

[50] P. Vashishta, K.S. Singwi, Phys. Rev. B 6, 875 (1972).

[51] J. Hubbard, Proc. R. Soc. (London) A 234, 336 (1957).

[52] S.S. Mandal, B.K. Rao, D.N. Tripathy, Phys. Rev. B 18, 2524 (1978).

[53] Z. Qian, Phys. Rev. B 73, 035106 (2006). 